\title{
Complex Network Analysis of Experimental EEG Signals for Decoding Brain Cognitive State
}

\author{
Zhongke Gao, Senior Member, IEEE, Zhu Gong, Qing Cai, Chao Ma, and Celso Grebogi
}

\begin{abstract}
Depicting the relationship between brain cognitive state and task difficulty level constitutes a challenging problem of significant importance. In order to probe it, we design an experiment to gather EEG data from mental arithmetic task under different difficulty levels. We construct brain complex networks using a complex network method and information entropy theory. We then employ weighted clustering coefficient to characterize the networks generated from different brain cognitive states. The results show that with the increase in task difficulty level, the mean weighted clustering coefficients show a decrease. This is due to the lack of coordination of brain activity and the low efficiency of the network organization caused by the increase in task difficulty. In addition, we calculate the permutation entropy from the signals of each channel EEG signals to support the findings from our network analysis. These findings render our method particularly useful for depicting the relationship between brain cognitive state and difficulty level.
\end{abstract}

Index Terms-Complex Network; Electroencephalogram (EEG); Brain Cognition; Time Series Analysis.

\section{INTRODUCTION}

$\mathbf{T}$ HE understanding of the brain lends itself as the leading transdisciplinary research effort in this century. To study some of its properties, researchers use different brain imaging techniques, for instance, functional magnetic resonance imaging (fMRI) [1], electroencephalography (EEG) [2], [3] and magnetoencephalography (MEG) [4]. As a temporal resolution brain electrical signals acquisition method, non-invasive and directly measurable, EEG is significantly more cost effective than most other techniques [5] and the size of its acquisition equipment is smaller than the others. Consequently, EEG is comprehensively used by researchers to monitor brain physiological activity. Among different areas of brain research, brain behavior dynamics greatly benefits from understanding the brain mechanism. Particularly, brain cognitive research, which is an approach of analyzing brain behavior dynamics, benefits from the research involving the working mechanism of the human brain. In this work, we aim at understanding an aspect of cognition by studying brain connectivity using the theory of complex networks. We investigate brain changes by designing cognitive experiments involving different cognitive difficulty levels. Cognitive difficulty level is the degree of mental effort to complete a cognitive task (e.g. mental arithmetic, problem

This work was supported by the National Natural Science Foundation of China under Grant Nos. 61903270, 61922062, 61873181. (Corresponding author: Chao Ma.)

Z. Gao, Z. Gong, Q. Cai, C. Ma are with the School of Electrical and Information Engineering, Tianjin University, Tianjin 300072, China (e-mail: chao.ma@tju.edu.cn).

C. Grebogi is with the Institute for Complex Systems and Mathematical Biology, King's College, University of Aberdeen, Aberdeen AB24 3UE, UK. solving, working memory, and reading). Cognitive difficulty level does impact on the amount of mental resources required by the subject to deal with a given cognitive task [6]. It is widely understood that the cognitive state changes with the change of brain activities [7]. But it is still not well understood how the brain cognitive state is affected by brain activities. Therefore, depicting changes of brain state under the task difficulty levels becomes an emerging hot research topic.

Previous researchers have published on the brain functional mechanisms under different cognitive states. Grundy et al. [8] used multiscale entropy analysis and multivariate statistics on EEG data to characterize the relationship between brain signal complexity and task difficulty. Sciaraffa et al. [9] used multiple-brain connectivity analysis to EEG data collected from a cooperative task and to reveal that the task difficulty level has impact on the averaged local properties of a brain network. Those papers discussed the relationship between brain state and task difficulty level from different angles. However, in order to reflect changes of brain cognitive state as a whole, to uncover the inherent non-linear characteristics of EEG signals, and the complex dynamic characteristics of the brain under different cognitive difficulty levels, a multidisciplinary approach using complex networks is called for.

For the past decades, complex network, as a complex system description method, has attracted much attention [10], [11]. Complex network provides new approaches of nonlinear time series analysis [12] and has been successfully used in network topology inference [13], multiphase flow [14], information propagation [15] and especially in brain science. A novel study [16], based on EEG signals, provides quantitative evidence that the disruption in the cortical inter-hemispheric connectivity and deficits in higher order cognitive functions are correlated with cognitive deficits. Another method for detecting epileptic seizure from EEG signals based on adaptive optimal kernel complex network was developed [17], achieving a highaccurate classification.

Many studies have shown that the brain can be thought of as a complex system [10], [18]. Different regions of the brain have their own distinct function, both simply and complex tasks require the cooperation and coordination of various brain regions, making the construction of functional brain network indispensable. In particular, the theory of multisource information fusion of complex network is applied increasingly to the study of brain cognitive state. For instance, EEG was used to detect the association with workload and mental states during cognitive tasks [19]. The description of EEG signal complexity can reflect the holistic state of brain function and reveal the inherent nonlinear characteristics of EEG signals. 
Therefore, it is of great significance to describe the complex dynamic characteristics of the brain under different cognitive difficulty levels by using, in particular, multiple EEG signals.

In this work, we design an experiment of mental arithmetics under different difficulty levels. Based on complex network method and information entropy theory, we combine topology information with functional connectivity information to develop a brain network construction method. We apply weighted clustering coefficient as a performance index to brain network analysis to reveal the complexity and the dynamic behavior of brain under different cognitive difficulty levels. The result shows that with the increase of cognitive difficulty levels, the mean weighted clustering coefficients show a decreasing tendency. This is due to the lack of coordination of brain activity and the low efficiency of network organization caused by the difficult tasks. These findings render our method particularly useful for depicting the interrelationship between brain cognitive state and difficulty level.

\section{PARTICIPANTS SELECTION AND EXPERIMENT DESIGN}

\section{A. Participants}

The 10 participants ( 7 males, 3 females, mean age 22.5 years) involved in this experiment are recruited from the School of Electrical and Information Engineering, Tianjin University. They all have normal or corrected-to-normal eyesight and are right-handed with no physiological or psychological medical history. Furthermore, the participants are required to abstain from alcohol, smoking, caffeine, tea and heavy meals for a minimum of one day before the experiment.

\section{B. Experimental Design}

The experiments are performed at the Laboratory of Complex Networks and Intelligent Systems, Tianjin University. We choose the arithmetic addition as the difficulty task for the experiment. The number of terms in the addition reflects the difficulty level of the task. During the experiment, a group of numbers is exhibited horizontally in the middle of a computer screen for the subjects to carry out the mental arithmetic addition. Three different difficulty levels correspond to three experimental conditions (addition of two numbers, six numbers and twelve numbers). Thirty random questions make up a sub-experiment, which includes ten questions (extracted randomly from a question bank) of each difficulty level. Each experiment is made up of three sub-experiments.

The stimulation interface of an experimental group: visual stimuli is displayed in the middle of the screen, four alternative answers are displayed below the question. The distance between the subjects' eyes and the screen is 50 centimeters. In addition, the eyes and the middle of the screen are aligned horizontally. When the mental arithmetic comes to a completion, the subject keys in the answer using the keyboard. The input answer is recorded in a text document. Correspondingly, the control group has no alternative answer, and the subjects click the mouse at the end of the response when the mental arithmetic finishes.

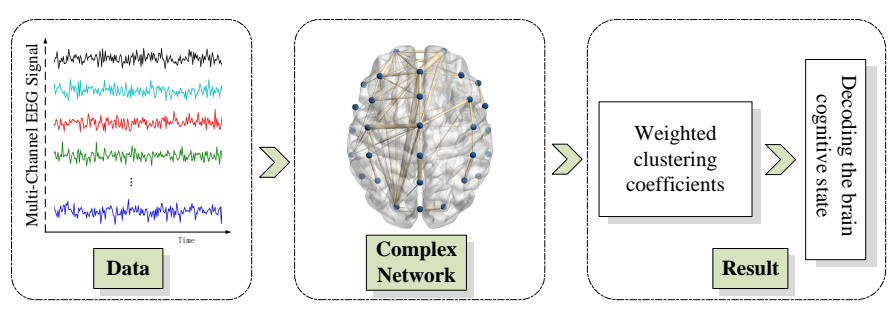

Fig. 1. Flow chart of complex network analysis.

\section{EEG Data Acquisition and Preprocessing}

The data acquisition process begins with the stimulation interface being presented, and ends up with all the questions being answered. The experiment interface is compiled by the software Eprime. EEG signals are collected by a 40channel NuAmps amplifier at $1000 \mathrm{~Hz}$ sampling rate, which confirms with the standard international 10-20 system. Before collection, EEG electrodes are covered with a conductive gel to require the skin impedance to be below $5 \mathrm{k} \Omega$. The equipped brain electrode cap includes 2 posterior auricular reference channels and 4 EOG acquisition channels (vertical and horizontal EOG channel), which are used for detecting eye blink and movement. We get 30 valid acquisition channels EEG signals.

EEGlab toolbox (Matlab) is used to preprocess the acquired EEG data, i.e., the acquired data is filtered with a bandpass of $1-40 \mathrm{~Hz}$ by finite impulse response (FIR) filter to eliminate noise. Subsequently, Independent Component Correlation Algorithm (ICA) is used to eliminate blink artifacts and EOG signal interference. Finally, the preprocessed data is organized by different difficulty levels to carry out the analysis.

\section{Methodology}

Based on the modelling method of Ref. [20], we combine topology information with functional connectivity information, based on a brain network construction method. The flow chart is shown in Fig. 1.

For a multi-channel signal $\left\{\mathrm{x}_{\mathrm{k}, \mathrm{j}}\right\}_{j=1}^{L}, k=1,2, \ldots, N$, containing $N$ sub-signals of equal length $L$, the Shannon entropy of any discrete sequence $i$ is defined as

$$
H_{I}=-\sum_{i} p(i) \log _{2} p(i),
$$

where $p(i)$ is the probability distribution of all states contained in the sequence $i$. Mutual information between a discrete sequence $i$ and a discrete sequence $j$ is defined as

$$
H_{I J}=-\sum_{i} p(i, j) \log \frac{p(i, j)}{p(i) p(j)}
$$

where

$$
M_{I J}=H_{I}+H_{J}-H_{I J} \geq 0 .
$$

Mutual information (MI), as an extension of information entropy, reflects the mutual dependence of two variables. That is to say, it measures the "amount of information" acquired about one random variable from detecting another 
random variable. MI has been successfully applied to time series analysis in many fields and it is symmetric under the interchange of $i$ and $j$, therefore MI does not carry direction information.

When constructing the brain network, in order to distinguish different frequency bands of EEG (delta, 2-4 Hz; theta, 4-8 $\mathrm{Hz}$; alpha1, 8-10 Hz; alpha2, 10-13 Hz; beta, 13-30 Hz; gamma, 30-50Hz), we use Fast Fourier Transform (FFT) to process the data. Then, for each frequency band, we consider the EEG channels as nodes of the network, and determine the edges as follows:

(1) Acquire edges with topology information. Locate the EEG channels, regard them as triangle vertices. The length of a triangle side is calculated by the physical distance. The triangle and its sides are considered to be available, only if the following conditions are satisfied: (a) there is no other vertices located in the triangle except the vertices on the sides; (b) the triangle is an acute triangle or a right triangle, i.e., it is not an obtuse triangle. If the triangle exists, set the edges to 1 , or else set it to 0 . Accordingly, we get a binary matrix $\boldsymbol{A}$.

(2) Acquire edges with functional connectivity information. Compute MI of every two EEG channels to obtain a 30 by 30 matrix. Delete proportional lowest edges of this matrix. In this work, we set the proportion as $30 \%-50 \%$ with an interval of $1 \%$ to get the weighted matrix $\boldsymbol{B}$.

(3) Combine topology information with functional connectivity information. If an edge is present both in matrix $\boldsymbol{A}$ and matrix $\boldsymbol{B}$, we then consider that this edge exists and assign its edge weight of matrix $\boldsymbol{B}$ to it. It still needs to meet the condition that for the triangle of every three nodes, the sum of any two sides is greater than the third side. In this way, we get the final adjacent matrix of our brain network.

These steps make up our brain network. We eliminate edges with less mutual dependence and avoid long-distant connections, which guarantee the effectiveness of our method from both information theory and topology points of view.

\section{DECODING BRAIN COGNITIVE STATE IN THE BRAIN NETWORK}

After constructing the brain network, we use clustering coefficient to characterize the brain network. In graph theory, a clustering coefficient is the index which measures the degree of nodes' tendency of clustering together [21]. Clustering coefficient is a reasonable measure of network functional segregation. There is evidences that nodes that are connected together tightly are often characterized by relatively intensive relation, which leads to larger clusters. The presence of clusters in functional networks suggests an organization of statistical dependencies indicative of segregated neural processing [22]. Thus, the mean weighted clustering coefficient for the network reflects the prevalence of clustered connectivity around an individual node. We use clustering coefficient as our brain network performance index to quantify the densely connected clusters of varied cognitive difficulty levels, in order to measure the dynamic functional segregation of the network. In weighted undirected network, clustering coefficient is defined as [23]

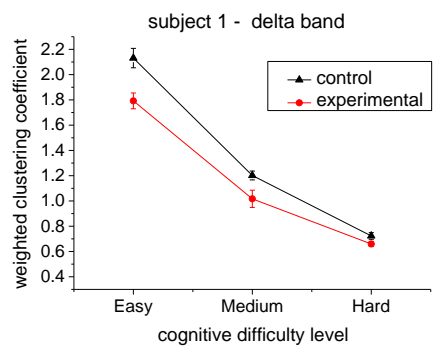

(a)

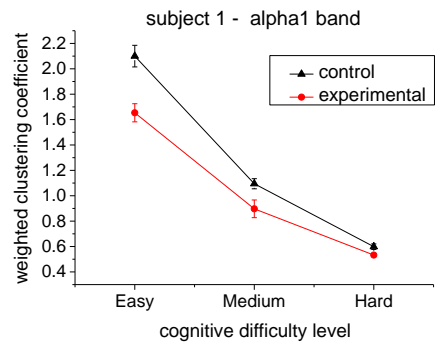

(c)

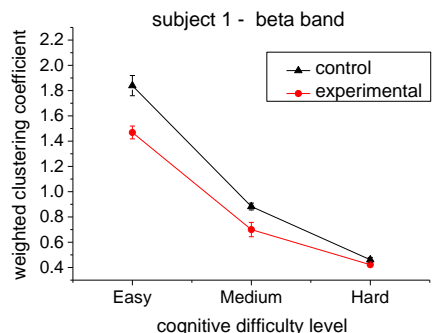

(e)

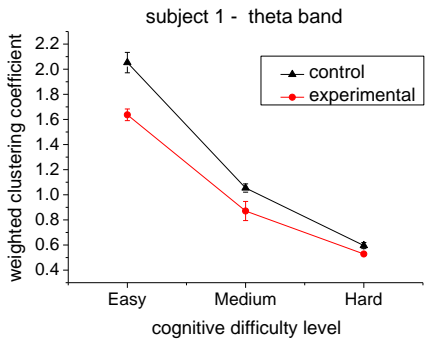

(b)

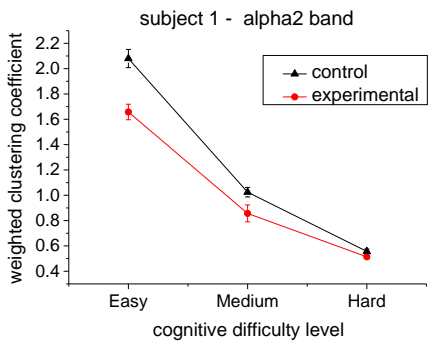

(d)

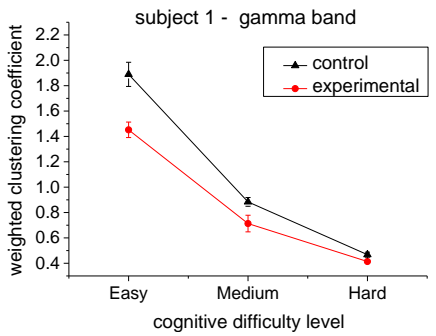

(f)
Fig. 2. Tendency between cognitive difficulty levels and brain network performance in experimental group (with alternatives, red circle) and control group (no alternative, black triangle) of a typical subject in different bands:(a) delta band; (b) theta band; (c) alpha1 band; (d) alpha2 band; (e) beta band; (f) gamma band.

$$
C^{w}=\frac{1}{n} \sum_{i \in N} C_{i}{ }^{w}=\frac{1}{n} \sum_{i \in N} \frac{2 t_{i}{ }^{w}}{k_{i}{ }^{w}\left(k_{i}{ }^{w}-1\right)},
$$

where $t_{i}{ }^{w}$ is the (weighted) geometric mean of triangles around node $i$, defined as

$$
t_{i}{ }^{w}=\frac{1}{2} \sum_{j, h \in N}\left(w_{i j} w_{i h} w_{j h}\right)^{\frac{1}{3}}
$$

and $k_{i}{ }^{w}$ is the weighted degree of node $i$, which is defined as

$$
k_{i}^{w}=\sum_{j \in N} w_{i j} .
$$

We calculate weighted clustering coefficient of brain network in different bands of all subjects. The result of a typical subject is presented in Fig.2, in which the horizontal axis shows the cognitive difficulty level of a task. Correspondingly, the vertical axis shows the mean values and the variances of weighted clustering coefficient of the brain network, revealing the clustering of the brain network. Red line shows experimental clustering coefficient with four alternatives in three difficulty levels, black line shows weighted clustering coefficient under experiment with no alternative in three difficulty levels. No matter which experimental group or control group, 
TABLE I

MEAN AND VARIANCE VALUES OF WEIGHTED CLUSTERING COEFFICIENT IN THE EXPERIMENTAL GROUPS OF ALL THE SUBJECTS IN THE BETA BAND.

\begin{tabular}{ccccccccc}
\hline \multirow{2}{*}{ Weighted CC } & \multicolumn{2}{c}{ Easy } & & \multicolumn{2}{c}{ Medium } & & \multicolumn{2}{c}{ Hard } \\
\cline { 2 - 3 } \cline { 7 - 8 } & Mean & Variance & & Mean & Variance & & Mean & Variance \\
\hline Subject 1 & 1.4686 & 0.0508 & & 0.7002 & 0.057 & & 0.4219 & 0.0094 \\
Subject 2 & 1.5304 & 0.0849 & & 0.969 & 0.0382 & & 0.7012 & 0.0403 \\
Subject 3 & 1.5005 & 0.1672 & & 0.9062 & 0.0517 & & 0.6414 & 0.0976 \\
Subject 4 & 1.8407 & 0.216 & & 1.2924 & 0.0389 & & 0.8711 & 0.01 \\
Subject 5 & 1.5745 & 0.0687 & & 0.8418 & 0.0342 & & 0.5605 & 0.0392 \\
Subject 6 & 1.8367 & 0.1037 & & 1.0588 & 0.0716 & & 0.6421 & 0.0753 \\
Subject 7 & 1.767 & 0.0763 & & 0.944 & 0.0771 & & 0.5796 & 0.0108 \\
Subject 8 & 1.7625 & 0.1665 & & 0.9613 & 0.0789 & & 0.5729 & 0.0171 \\
Subject 9 & 1.7174 & 0.0918 & & 1.0041 & 0.0335 & & 0.6048 & 0.0225 \\
Subject 10 & 1.6614 & 0.0371 & & 1.0661 & 0.1061 & & 0.5362 & 0.0247 \\
\hline
\end{tabular}

TABLE II

MEAN AND VARIANCE VALUES OF THE WEIGHTED CLUSTERING COEFFICIENT IN THE CONTROL GROUP OF ALL SUBJECTS IN BETA BAND.

\begin{tabular}{|c|c|c|c|c|c|c|}
\hline \multirow{2}{*}{ Weighted CC } & \multicolumn{2}{|c|}{ Easy } & \multicolumn{2}{|c|}{ Medium } & \multicolumn{2}{|c|}{ Hard } \\
\hline & Mean & Variance & Mean & Variance & Mean & Variance \\
\hline Subject 1 & 1.8394 & 0.0801 & 0.8827 & 0.0269 & 0.4613 & 0.0185 \\
\hline Subject 2 & 1.6586 & 0.0736 & 1.1504 & 0.0375 & 0.7542 & 0.0111 \\
\hline Subject 3 & 1.7229 & 0.1209 & 0.9535 & 0.083 & 0.5238 & 0.0181 \\
\hline Subject 4 & 1.9923 & 0.0989 & 1.3484 & 0.0825 & 1.0186 & 0.0179 \\
\hline Subject 5 & 1.8478 & 0.0558 & 0.9858 & 0.0481 & 0.5884 & 0.0215 \\
\hline Subject 6 & 2.017 & 0.1903 & 1.2447 & 0.0391 & 0.7228 & 0.0178 \\
\hline Subject 7 & 1.9065 & 0.1176 & 1.1262 & 0.1131 & 0.6106 & 0.0223 \\
\hline Subject 8 & 2.1603 & 0.1932 & 1.18 & 0.0999 & 0.6073 & 0.0408 \\
\hline Subject 9 & 2.0162 & 0.1063 & 1.0973 & 0.0587 & 0.7585 & 0.0705 \\
\hline Subject 10 & 1.7634 & 0.1458 & 1.2628 & 0.0748 & 0.5541 & 0.0118 \\
\hline
\end{tabular}

and what band is being considered, transition from easy to hard condition gives rise to decrease of the weighted clustering coefficient, which means that the clustering in brain network weakens gradually. By the same token, data of all ten subjects in all bands has the same tendency, which can be seen in Table I (experimental group) and Table II (control group).

The workload is positively related to cognitive difficulty level, which means that workloads increase as the cognitive task becomes more difficult [24]. Whether the experimental group or the control group, when the cognitive difficulty level is easy, the cognitive workload is relatively low, and the weighted clustering coefficient of the network has larger mean value and more significant variance. This is because the easy task involves fewer parts of the brain and the brain regions form clusters, bringing more efficient organization of the brain network, segregating brain functional activities. Besides, the addition of two terms can easily affect the task difficulty, leading to a larger variance. As the cognitive difficulty level and the cognitive workloads increase, the mean value of the weighted clustering coefficient of the network shows a decreasing tendency and variance that is not so obvious any longer. In order to carry out the task better, all parts of the brain are involved, clusters decrease, leading to a decreased brain functional segregation. Hard cognitive difficulty level gives rise to less coordinated brain activities and less efficient organization of the brain network. So the variance fluctuation is reduced. Shen et al. [24] and Sciaraffa et al. [9] also give their analysis to this phenomenon. When the number of terms adds up to 12 , reaching the highest cognitive difficulty level,

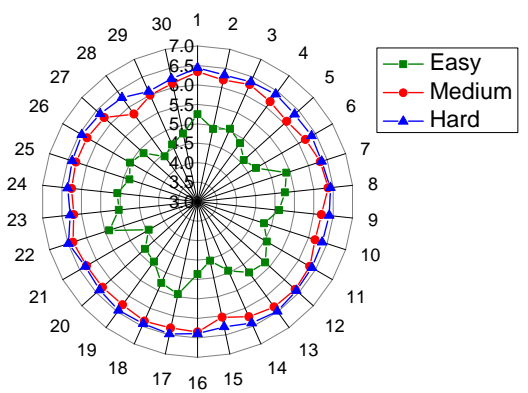

Fig. 3. A radar map of the permutation entropy distribution under three cognitive difficulty levels (Green: easy; Red: medium; Blue: hard) of a typical subject.

the weighted clustering coefficient of the network has the lowest mean value and the smallest variance, which means the worst functional segregation and the least efficient organization of the brain network. Meanwhile, no matter what difficulty level is, the control group has larger clustering coefficient than the experimental group. This can be partly explained by the fact that typing in the answers with keyboard needs more attention resulting in a more difficult cognition. The validity of our results is further verified by it.

In order to better characterize the complexity of the brain, we compute the permutation entropy of 30-channel EEG signals under three different cognitive difficulty levels from all subjects. The distribution of permutation entropy of a typical subject is presented in the form of a radar map in Fig. 3. Permutation entropy is a nonlinear complexity measure for time series, with the advantages of simplicity, extremely fast calculation, robustness, and invariance with respect to nonlinear monotonous transformations [25]. For any time series $x(t), t=1,2, \ldots$, embed it into an $\mathrm{m}$-dimensional space

$$
X(t)=[x(t), x(t+\tau), \ldots, x(t+m \tau)],
$$

where $m$ is the embedding dimension and $\tau$ is the time lag. For a given embedding dimension, there are $m$ ! possible permutations. If each permutation is considered as a symbol, the embedded time vector $X(t)$ can be represented by a symbol sequence $j$, each having probability distribution $p_{j}$. Thus, based on the Shannon entropy definition, the permutation entropy $H_{p}$, is defined as

$$
H_{p}(m)=-\sum_{j=1}^{k} p_{j} \ln \left(p_{j}\right) .
$$

When $p_{j}=\frac{1}{m !}, H_{p}$ reaches maximum $\ln (m !)$. The changes of $H_{p}$ reflect and amplify the subtle changes in the time series, while the value of $H_{p}$ represents the degree of randomness; a smaller permutation entropy means a more regular time series [26].

In the easy task, the permutation entropy is relatively small and there are differences among the channels, indicating that the complexity and nonlinearity are relatively low but slightly uneven distribution. With the cognitive difficulty level increased, the permutation entropy increases as well, showing higher complexity and nonlinearity. Besides, the permutation 
entropy among the channels shows relatively even distribution under medium and hard task. Permutation entropy analysis demonstrates the increase in complexity and nonlinearity when the cognitive difficulty level increases, which supports our results and indicate their validity. Though we can spot the difference of the permutation entropy between medium and hard tasks, however, the values are still too close to distinguish.

\section{CONCLUSION}

In this paper, we articulate a strategy for decoding brain cognitive states under different difficulty levels from EEG signals. First, we design an experiment of mental arithmetic under three difficulty levels and collect the EEG data. We regard each channel as a node in a complex network. We combine topology and mutual information from information theory to obtain the edges. Finally, we compute mean values and variances of weighted clustering coefficients of networks under different cognitive difficulty levels to characterize functional segregation of the brain network. The results show that with the increase of cognitive difficulty level, the mean weighted clustering coefficient presents a decreasing tendency, which is due to the lack of coordination of brain activity and the low efficiency of network organization caused by the difficult tasks. Resulting from no alternative as a reference also promotes the difficulty, the mean weighted clustering coefficients of the control group under the same cognitive difficulty level are lower than that of the experimental group. At the same time, the variances of the weighted clustering coefficient decrease, which could be accounted by the fluctuation among each tasks of the same cognitive difficulty level. In addition, we also compute the permutation entropy in order to analyze EEG signals under different cognitive difficulty levels, and found that the medium and difficult tasks are indeed more complex than the easy task. This validates the validity of our experimental design. Meanwhile, the value of permutation entropy under medium and hard tasks are extremely close, thus the permutation entropy method is hard to make the distinction, which proves the necessity of our experiment. Our results demonstrate that our methods are capable of accurately reflecting changes in tendency of the brain network under various experimental conditions by decoding brain cognitive state.

\section{REFERENCES}

[1] D. Scheinost, F. Tokoglu, X. Shen, E. S. Finn, S. Noble, X. Papademetris, and R. T. Constable, "Fluctuations in global brain activity are associated with changes in whole-brain connectivity of functional networks," IEEE Trans Biomed Eng, vol. 63, no. 12, pp. 2540-2549, Dec. 2016.

[2] Z. Gao et al., "A deep learning method for improving the classification accuracy of SSMVEP-based BCI," IEEE Trans Circuits Syst II, to be published. DOI: 10.1109/TCSII.2020.2983389

[3] Z. Gao et al., "EEG-based spatio-temporal convolutional neural network for driver fatigue evaluation," IEEE Trans Neural Netw Learn Syst, vol. 30, no.9, pp.2755-2763, Sep. 2019.

[4] J. Zhang et al., "Decoding Brain States Based on Magnetoencephalography From Prespecified Cortical Regions," IEEE Trans Biomed Eng, vol. 63, no. 1, pp. 30-42, Jan. 2016.

[5] P. M. Vespa, V. Nenov, and M. R. Nuwer, "Continuous EEG monitoring in the intensive care unit: Early findings and clinical efficacy," J Clin Neurophysiol, vol. 16, no. 1, pp. 1-13, Jan. 1999.
[6] S. C. Fish and E. Granholm, "Easier tasks can have higher processing loads: Task difficulty and cognitive resource limitations in schizophrenia," J Abnorm Psychol, vol. 117, no. 2, pp. 355-363, May 2008.

[7] E. Galy, M. Cariou, and C. Melan, "What is the relationship between mental workload factors and cognitive load types?" Int J Psychophysiol, vol. 83, no. 3, pp. 269-75, Mar. 2012.

[8] J. G. Grundy, R. M. Barker, J. A. E. Anderson, and J. M. Shedden, "The relation between brain signal complexity and task difficulty on an executive function task," Neuroimage, vol. 198, pp. 104-113, Sep. 2019.

[9] N. Sciaraff, G. Borghini, P. Arico, G. D. Flumeri, A. Colosimo, A. Bezerianos, N. V.Thakor, and F. Babiloni, "Brain interaction during cooperation: Evaluating local properties of multiple-Brain network," Brain sci, vol. 7, no. 7, Jul. 2017.

[10] E. Bullmore and O. Sporns, "Complex brain networks: graph theoretical analysis of structural and functional systems," Nat Rev Neurosci, vol. 10, no. 3, pp. 186-98, Mar. 2009.

[11] S. Boccaletti, V. Latora, Y. Moreno, M. Chavez, and D. Hwang, "Complex networks: Structure and dynamics," Phys Rep, vol. 424, no. 4-5, pp. 175-308, 2006.

[12] Z. Gao, M. Small, and J. Kurths, "Complex network analysis of time series," EPL, vol. 116, no. 5, p.50001, Dec. 2016.

[13] J. Zou, X. Yu, J. Lu, "Node importance in controlled complex networks," IEEE Trans Circuits Syst II, vol. 66, no. 3, pp. 437-441, Mar. 2019.

[14] Z. Gao, W. Dang, C. Mu, Y. Yang, S. Li, and C. Grebogi, "A novel multiplex network-based sensor information fusion model and its application to industrial multiphase flow system," IEEE Trans Industr Inform, vol. 14, no. 9, pp. 3982-3988, Sep. 2018.

[15] M. Ogura, W. Mei, and K. Sugimoto, "Synergistic effects in networked epidemic spreading dynamics," IEEE Trans Circuits Syst II, vol. 67, no. 3, pp. 496-500, Mar. 2020.

[16] L. Gao, C. Grebogi, Y. Lai, J.Stephen, T. Zhang, Y. Li, H. Ren, D. Li, J. Wang, B. Schelter, and L. Sommerlade, "Quantitative assessment of cerebral connectivity deficiency and cognitive impairment in children with prenatal alcohol exposure," Chaos, vol. 29, no. 4, pp. 041101, Apr. 2019.

[17] Z. Gao, Q. Cai, Y. Yang, N. Dong, and S. Zhang, "Visibility graph from adaptive optimal kernel time-frequency representation for classification of epileptiform EEG,' Int J Neural Syst, vol. 27, no. 4, p. 1750005, Jun. 2017.

[18] O. Sporns, D. R. Chialvo, M. Kaiser, and C. C. Hilgetag, "Organization, development and function of complex brain networks," Trends Cogn Sci, vol. 8, no. 9, pp. 418-425, Sep. 2004.

[19] G. Borghini, L. Astolfi, G. Vecchiato, D. Mattia, and F. Babiloni, "Measuring neurophysiological signals in aircraft pilots and car drivers for the assessment of mental workload, fatigue and drowsiness,"Neurosci Biobehav Rev, vol. 44, pp. 58-75, Jul. 2014.

[20] G. Li, B. Li, Y. Jiang, W. Jiao, H. Lan, and C. Zhu, "A new method for automatically modelling brain functional networks," Biomed Signal Process Control, vol. 45, pp. 70-79, Aug. 2018.

[21] G. Fagiolo, "Clustering in complex directed networks," Phys Rev E, vol. 76, no. 2, p. 026107, Aug. 2007.

[22] M. Rubinov and O. Sporns, "Complex network measures of brain connectivity: uses and interpretations," Neuroimage, vol. 52, no. 3, pp. 1059-69, Sep. 2010.

[23] J. P. Onnela, J. Saramaki, J. Kertesz, and K. Kaski, "Intensity and coherence of motifs in weighted complex networks," Phys Rev E, vol. 71, no. 6, p. 065103, Jun. 2005.

[24] R. Shen, L. Junhua, F. Taya, J. deSouza, N. V. Thakor, and A. Bezerianos, "Dynamic functional segregation and integration in human brain network during complex tasks," IEEE Trans Neural Syst Rehabil Eng, vol. 25, no. 6, pp. 547-556, Jun. 2017.

[25] C. Bandt and B. Pompe, "Permutation entropy: a natural complexity measure for time series," Phys Rev Lett, vol. 88, no. 17, p. 174102, Apr. 2002.

[26] Y. Cao, W. Tung, J. Gao, V. A. Protopopescu, and L. M. Hively, "Detecting dynamical changes in time series using the permutation entropy," Phys Rev E, vol. 70, no. 4, p. 046217, Oct. 2004. 